\title{
Breast Cancer Treatment in the Era of Precision Medicine
}

\author{
Marcus Vetter ${ }^{1}$, Christian Kurzeder ${ }^{1}$
}

ABSTRACT

Breast cancer is the most common cancer in women in the Western world. Treatment decisions in the adjuvant and metastatic setting are based on three immunohistochemistry markers, namely estrogen receptor (ER), progesterone receptor (PR), and human epidermal growth factor receptor 2 (HER2). Based on the St. Gallen consensus, four distinct subtypes are defined: luminal A, luminal B, HER2-enriched and basal-like subtype breast cancer. In the era of precision medicine, more accurate profiling of breast cancer is now possible. Highly specific methods, including, but not limited to, next-generation sequencing (NGS), proteomics, and immune profiling, help to identify the targets for the appropriate treatment. Several international consortia trials, e.g., AURORA from the International Breast Cancer Study Group (IBCSG), are on the way to standardize optimal molecular treatment for breast cancer patients. This review gives you a clinically oriented overview of treatment for all breast cancer subtypes and an insight into molecular techniques and profile-based decisions. It is an overview from clinicians for clinicians.

Keywords: breast cancer, molecular subtypes, targeted therapies, endocrine therapy, prognostic markers, precision medicine

\section{INTRODUCTION}

Worldwide, breast cancer is the most common cancer in females, with an annual incidence rate of $80-113$ cases per 100,000 women. ${ }^{1}$ The incidence has increased through recent decades, but the mortality rate has steadily dropped over the last 30 years. ${ }^{2}$ Rising incidence is attributed to a combination of better diagnostic methods, lifestyle changes, hormonal replacement therapies, improved screening initiatives, and earlier detection. ${ }^{3}$ The declining mortality rate is also associated with screening, together with modernized treatment options, including surgery, chemotherapy, targeted therapy, and radiotherapy. Today, $85 \%$ of patients with a breast cancer diagnosis can be cured with multimodal therapy. ${ }_{4}^{4}$ However, while the aim of treatment is to cure, quality of life and prevention of acute and late treatment-associated toxicity is paramount to the decision process. ${ }^{5}$

The St. Gallen Consensus defines four clinically relevant subtypes of breast cancer: luminal A, luminal B, human epidermal growth factor receptor 2 (HER2)-enriched, and triple-negative disease (TNBC) subtypes (Table 1). This basic classification is predominantly based on immunochemistry (IHC) markers, including estrogen receptor (ER), progesterone receptor (PR), ERBB2/HER2, and Ki-67. These clinically established, yet simple targets generally correlate with the intrinsic subtypes and still help to guide treatment decisions today. However, through whole-genome sequencing, single-cell analysis, and proteomics, new targets are on the horizon to improve treatment decision making and outcomes. ${ }^{6}$

In the era of precision medicine, it is generally accepted that breast cancer is characterized beyond ER/PR/HER2; each subtype requires specific treatment regimens, thus necessitating the evolution of the breast cancer treatment landscape. Recent data from the Molecular Taxonomy of Breast Cancer International Consortium (METABRIC) Study demonstrated
${ }^{1}$ Breast Cancer Center, University Hospital Basel, Spitalstrasse 21, 4031 Basel, Switzerland

\section{Correspondence:}

PD Dr med. Marcus Vetter

Medical Oncology \& Breast Cancer

Center

University Hospital Basel

Spitalstrasse 21

4031 Basel

Phone: +41-61 2652525

Email:marcus.vetter@usb.ch

DOI:10.36000/hbT.OH.2020.05.020 ISSN: 26/3-2092 (Print) and 26/32106 (Online)

The article was published on 12.10.2020

Vetter $\mathrm{M}$ et al. Breast Cancer Treatment in the Era of Precision Medicine. healthbook TIMES Onco Hema 2020;(5):26-37 


\begin{tabular}{|l|l|l|l|l|}
\hline Subtype & ER & PR & KI-67 & ERBB2/HER2 \\
\hline Luminal A & + & + & $<15 \%$ (low) & - \\
Luminal B & + & + & $>15 \%$ (high) & - \\
HER2-enriched & $+/-$ & $+/-$ & Both & + \\
Triple negative & - & - & High & - \\
\hline
\end{tabular}

Table 1. Breast Cancer subtypes

in accordance to St. Gallen

Consensus. ${ }^{115} \mathrm{ER}$, estrogen

receptor; HER2, human epidermal growth factor receptor $2, \mathrm{PR}$, progesterone receptor.
10 molecular subtypes with distinct, yet comparable, clinical outcomes. ${ }^{7}$ This group performed an integrated analysis of copy number and gene expression in a discovery group of 997 primary breast tumors, and a subsequent validation set of 995 samples, to discern reproducibility, with long-term clinical follow-up.

In general, for HER2-positive and TNBC disease, neoadjuvant systemic therapy is applied before surgery. ${ }^{8}$ The pathological complete response $(\mathrm{pCR})$ rate in TNBC patients has increased through the use of immune checkpoint inhibitors plus standard chemotherapy.9 In HER2-positive disease, dual inhibition of the HER2 kinase with trastuzumab and pertuzumab plus chemotherapy increased $\mathrm{PCR}$ rate and eventfree survival. ${ }^{10,11}$ Adjuvant trastuzumab emtansine is another new strategy for patients with HER2-positive disease who do not achieve $\mathrm{pCR} .^{12}$

In luminal $\mathrm{A} / \mathrm{B}$ disease, about $25 \%$ of patients receive chemotherapy after primary surgery. Genetic signatures like the Oncotype DX ${ }^{\circ}$, MammaPrint ${ }^{\circ}$, EndoPredict ${ }^{\circ}$, Predictor of Microarray 50 (PAM50), and others help to identify tumors that can potentially benefit from additional chemotherapy. ${ }^{13-16}$ Today, chemotherapy is still based on anthracycline and taxanes regimens. ${ }^{8}$ A recent randomized study, which included over 10,000 women with hormone receptor-positive/ HER2-negative breast cancer, demonstrated the beneficial role of chemotherapy in patients with intermediate or highrisk disease, with certain risk factors, including early age, menopausal status and midrange 21 -gene recurrence score. ${ }^{13}$

By shortening the intervals between therapy cycles or delivering individual drugs at full-dose sequentially, the intensity of cytotoxic therapy can be increased with relative safety. This dose-dense concept is becoming increasingly important, especially in patients with high-risk tumors, e.g., high grading, increased lymph node involvement, and high proliferation. ${ }^{17-20}$ These drug delivery methods have shown improved relapse-free survival. A recent meta-analysis from the Oxford-Overview group clearly emphasizes the role of a dosedense regimen for improved clinical outcomes. ${ }^{18}$ On the other hand, the potential long-term toxicities, such as hematologic malignancy, heart and lung disease, as well as clinical benefits, should be considered in patient's informed consent. ${ }^{21-23}$

\section{TUMOR HETEROGENEITY IN THE ERA OF PRECISION MEDICINE}

Relapse will occur in around $20 \%$ of breast cancer patients. ${ }^{1}$ The most relevant relapse risk factors are grading, disease stage, and breast cancer subtype, including TNBC and HER2positive disease. ${ }^{24,25} \mathrm{~A}$ recently published overview of luminal breast cancer, with a very long follow-up of 20 years after treatment cessation, showed a relapse rate of more than $40 \%$ in patients with a high number of lymph nodes over a follow-up time of 25 years after initial diagnosis. ${ }^{26}$ Tumor heterogeneity might be the most important reason for early and later relapse in all breast cancer subtypes. ${ }^{27}$ In addition to heterogeneous ER, PR, and HER2 expression among patients with breast cancer, different expression levels between primary cancer and matched metastasis has been observed. ${ }^{28}$ Nowadays, new techniques like gene-expression profiling or massively parallel sequencing have helped to identify the genetic background of primary and metastatic breast cancers. ${ }^{29,30}$

Based on a recently published meta-analysis, there is a wide range of expression levels for ER, PR, and HER2 in primary and metastatic breast cancer. ${ }^{31}$ In this analysis, more the 4,000 tumors were examined. Pooled proportions of tumors shifting from positive to negative and from negative to positive were $24 \%$ and $14 \%$ for ER ( $p=0.0183$ ), $46 \%$ and $15 \%$ for PR $(p<0.0001)$, and $13 \%$ and $5 \%$ for HER2 $(p=0.0004)$. The change of expression profile has a major implication on treatment selection, and biopsy of the metastatic site is strongly recommended in patients with relapsed metastatic breast cancer.

Based on current research, there are different types of tumor-associated heterogeneity, including intertumoral and intratumoral heterogeneity. ${ }^{7}$ The management of intertumoral heterogeneity patients and tumors should include patient stratification based on molecular profiling. In addition, treatment trials need to have innovative designs, including master protocols, basket trials, and adaptive trial design. For a better characterization of intratumoral heterogeneity, a 


\section{healthbook TIMES Oncology Hematology}

metastatic biopsy is necessary to determine the profile of the tumor. Furthermore, a longitudinal tumor follow-up, including repeated metastatic biopsy is necessary. Finally, techniques like NGS, bioinformatics tools and animal models (PDX models) should be used in order to identify driverevents and mutations. ${ }^{28}$

\section{MOLECULAR SUBTYPES OF BREAST CANCER AND PRECISION MEDICINE (ADVANCED BREAST CANCER)}

Immune checkpoint blockade and combinations in TNBC Triple-negative breast cancer (TNBC), a heterogeneous breast cancer subgroup, is defined by the absence of detectable ER, HER, and PR expression. ${ }^{32-34}$ In 2011, Lehmann et al. defined 6 subgroups of TNBC with different outcomes and therapeutic implications (Table 2). ${ }^{34}$ TNBC is associated with poor clinical course, younger age, and hereditary cancers when compared with HER2-positive and ER-positive breast cancers. There is also an association of TNBC and BRCA1/2 mutations and homologous recombination deficiency (HRD). ${ }^{35}$ Patients with TNBC frequently have a poor prognosis with a relapse rate of $30 \%$ in the primary setting. In the last decades, fewer new drugs have been approved in the metastatic setting.

Considering the molecular landscape of TNBC, more than $90 \%$ of tumors have molecular alterations in at least one signaling pathway. These include PIK3CA/AKT/mTOR ( $\sim 0 \%$ ), cell-cycle pathways such as RB1, CDK2, 4, 6, and others (40\%) and RAS/MAP-kinase pathway ( 12\%). In addition, overexpression of growth factor receptors (GFR) including EGFR, MET, and EGF1R ( 15\%) and deficiencies in DNA repair including BRCA $(\sim 12 \%)$ has been reported in TNBC tumors. ${ }^{36}$

Besides targeting molecular alteration, immunotherapy has emerged as another important treatment strategy. ${ }^{37,38}$ So far, the current treatment option with a single-agent immune checkpoint inhibitor showed modest treatment responses. The KEYNOTE-012 study was a phase Ib study that demonstrated an objective response rate (ORR) of $18.5 \%$ in heavily pretreated TNBC who were programmed deathligand 1 (PD-L1)-positive $(\mathrm{n}=32) \cdot{ }^{39}$ Further research showed an important role of tumor-infiltrating lymphocytes (TIL) and programmed cell death protein 1 (PD1)/ PD-L1 status as an important biomarker in the metastatic setting (Table 3).

In 2019, the TONIC trial evaluated several combination strategies with nivolumab, a programmed cell death protein 1 (PD-1) inhibitor. ${ }^{40}$ In this study, 67 patients underwent randomization to receive either nivolumab as monotherapy or in combination with a) irradiation, b) chemotherapy with doxorubicin, c) low-dose cyclophosphamide, or d) chemotherapy with cisplatin, all followed by nivolumab maintenance therapy. The highest response rates were reported in the doxorubicin (23\%) and cisplatin (35\%) cohorts. After doxorubicin and cisplatin induction, the upregulation of genes involved in PD-1/PD-L1 and T-cell cytotoxicity pathways was observed. Results further showed upregulation of JAKSTAT and TNF- $\alpha$ signaling in the doxorubicin cohort and combinations with this mechanism might be promising for further evaluation. The trial concluded that the combination of doxorubicin/cisplatin and immunotherapy with nivolumab might induce a more favorable tumor environment and increase the likelihood of response to PD-1 blockade. Further analysis and clinical trials are needed to define the best combination regimes.

IMpassion130, a landmark trial published in the New England Journal of Medicine, showed that a combination of nab-paclitaxel and atezolizumab, a PD-1 inhibitor, provide an overall survival (OS) benefit among TNBC patients with PD-L1-positive tumors (the expression on tumor-infiltrating immune cells $\geq 1 \%$ ) compared with single-agent nab-

Table 2. Triple-negative breast cancer, subtypes based on Lehmann et al. ${ }^{34} \mathrm{AR}$, androgen receptor; PARP, poly (ADP-ribose) polymerase.

\begin{tabular}{|c|c|c|}
\hline Subtype & Alterations/pathways & Therapeutic target/drug \\
\hline Basal-1 & $\begin{array}{l}\text { Cell cycle, DNA replication, G2 pathway, RNA polymerase, ATR/ } \\
\text { BRCA pathway, G1 to S cell cycles }\end{array}$ & Platinum, PARP-inhibitors, and others \\
\hline Basal-2 & EGF pathway, NGF pathway, MET pathway, and others & $\begin{array}{l}\text { Platinum, PARP-inhibitors, } \\
\text { met-inhibitors, and others }\end{array}$ \\
\hline Immunomodulatory & $\begin{array}{l}\text { CTLA4 pathway, IL12 pathway, NK cell pathway, TH1/TH2 } \\
\text { pathway, T-cell signaling, and others }\end{array}$ & Immune-check-point-inhibitors and others \\
\hline Mesenchymal-like & IGF/mTOR pathway, ECM pathway, WNT pathway, and others & TKI, mTOR-inhibitors, eribulin, and others \\
\hline Mesenchymal Stem-like & $\begin{array}{l}\text { ECM-receptor interaction, TCR pathway, WNT pathway, focal } \\
\text { adhesion, NK-cell mediated toxicity, GH pathway, and others }\end{array}$ & Similar to $\mathrm{M}$ growth factor signaling \\
\hline Luminal-AR & $\begin{array}{l}\text { Pentose/glucuronate interconversion, tyrosine metabolism, citrate } \\
\text { cycle TCA and others }\end{array}$ & $\begin{array}{l}\text { AR-receptor signaling: AR-inhibitors and } \\
\text { others }\end{array}$ \\
\hline
\end{tabular}

healthbook Times Oncology Hematology healthbook.ch October, 2020 
Table 3. Clinical trials with monotherapy with an immune-check-point-inhibitor in metastatic TNBC. ORR, overall response rate; mPFS, median progressionfree-survival; mOS, median overall survival; M, months; PD-L1, programmed death-ligand 1.

\begin{tabular}{|c|c|c|c|c|c|c|c|c|}
\hline Trial & Therapy & $\mathrm{N}$ of lines & PD-L1 & $\mathrm{N}$ & $\begin{array}{c}\text { ORR } \\
(\%)\end{array}$ & mPFS, (M) & mOS, (M) & Literature \\
\hline Keynote-012 & Pembrolizumab & $\begin{array}{c}\text { Median } 2 \\
(0-9)\end{array}$ & + & 32 & 18.5 & 1.9 & 11.2 & 116 \\
\hline Keynote-086A & Pembrolizumab & $\geq 1$ & $\begin{array}{c}+/- \\
+ \\
-\end{array}$ & $\begin{array}{c}170 \\
105 \\
64\end{array}$ & $\begin{array}{l}5.3 \\
5.7 \\
4.7\end{array}$ & $\begin{array}{l}2.0 \\
2.0 \\
1.9\end{array}$ & $\begin{array}{l}9.0 \\
8.8 \\
9.7\end{array}$ & 117 \\
\hline JAVELIN & Avelumab & $\begin{array}{c}\text { Median } 2 \\
(1-6)\end{array}$ & $\begin{array}{c}+/- \\
+ \\
-\end{array}$ & $\begin{array}{c}58 \\
9 \\
39\end{array}$ & $\begin{array}{r}5.2 \\
22.2 \\
2.6\end{array}$ & 5.9 & 9.2 & 118 \\
\hline NCT01375842 & Atezolizumab & $58 \% \geq 2$ & $78 \%+$ & 115 & 10 & 1.4 & 8.9 & 119 \\
\hline
\end{tabular}

paclitaxel (25.0 months vs 15.5 months; HR: 0.62 [95\% CI: $0.45-0.86]) .^{41}$ These data also indicated that the biomarkerderived approach and the combination of chemotherapy and immunotherapy is an important treatment strategy in this patient population. ${ }^{42,43}$ As a result, this drug combination has become the standard of care for metastatic PD-L1-positive TNBC. However, atezolizumab plus paclitaxel recently failed to show an improvement in progression-free survival (PFS) compared with paclitaxel alone in $\mathrm{PD}-\mathrm{L} 1$ positive patients in the IMpassion 131 trial. Furthermore, a negative trend for OS was observed, although data are immature and not sufficiently powered. ${ }^{44}$ Future perspectives include a combination strategy of different immune therapy agents and chemotherapy.

Currently, a large phase III trial is evaluating the combination of chemotherapy, immunotherapy and targeted therapy with ipatasertib, a novel small molecule inhibiting AKT, an important component of the cancer pathway PIK3CA.45 Previously, in preclinical models, ipatasertib demonstrated activity against all three isoforms of AKT. ${ }^{46}$ Furthermore, in a first-in-human phase I study, ipatasertib monotherapy showed clinically meaningful tumor control (disease control rate of $30 \%$ [16/52 patients]) and manageable toxicity in patients with solid tumors (median prior lines of treatment: $6[1-17])$, including breast (31\%), colorectal (27\%), prostate (12\%) chondrosarcoma (4\%), ovarian (4\%) and other (22\%) tumors. ${ }^{47}$ The most common toxicity was gastrointestinal toxicity of grade $1-2$.

Ipatasertib was also assessed in patients with TNBC. The LOTUS trial investigated the addition of ipatasertib to paclitaxel as first-line therapy in patient with TNBC. ${ }^{48}$ In this phase II trial, 124 patients with advanced metastatic TNBC received paclitaxel $\left(80 \mathrm{mg} / \mathrm{m}^{2}\right.$ on days $\left.1,8,15\right)$, with or without ipatasertib (400 mg on days 1-21). PTEN status was assessed in all patients. The co-primary endpoints, PFS in the intention-to-treat population, and PFS in the PTENlow (by IHC) patients, were 6.2 months versus 3.9 months, respectively (HR: 0.60 [95\% CI: 0.37-0.98]; p=0.037). Further evaluation in TNBC was recommended by the authors. Ipatasertib has been further evaluated with a modern anti-PD-L1 inhibitor and chemotherapy (paclitaxel weekly) within the IPATUNITY studies. ${ }^{49}$ Preliminary results from this phase Ib study demonstrated that a combination of ipatasertib plus atezolizumab and paclitaxel/nab-paclitaxel provided a response rate of $73 \%$ in patients with locally advanced/metastatic TNBC, irrespective of biomarker status, with a manageable toxicity profile.

\section{HER2-directed therapy in advanced HER2-positive} breast cancer

HER2-positive breast cancer accounts for up to $15 \%$ of breast cancer and is characterized by the overexpression of the receptor tyrosine kinase HER2. ${ }^{50}$ In general, the prognosis in this breast cancer subtype is poor, with an aggressive course of the disease. ${ }^{51}$ However, several new systemic treatment options have improved survival outcomes over the last two decades. For example, trastuzumab has provided a significant clinical benefit in patients with metastatic HER2-positive breast cancer, which led to the approval of this agent for the treatment of this patient population more than 18 years ago. ${ }^{52,53}$ In addition, trastuzumab, the current standard of care, is associated with improved clinical outcomes in the adjuvant setting. ${ }^{8}$ Within the last five years, several new 


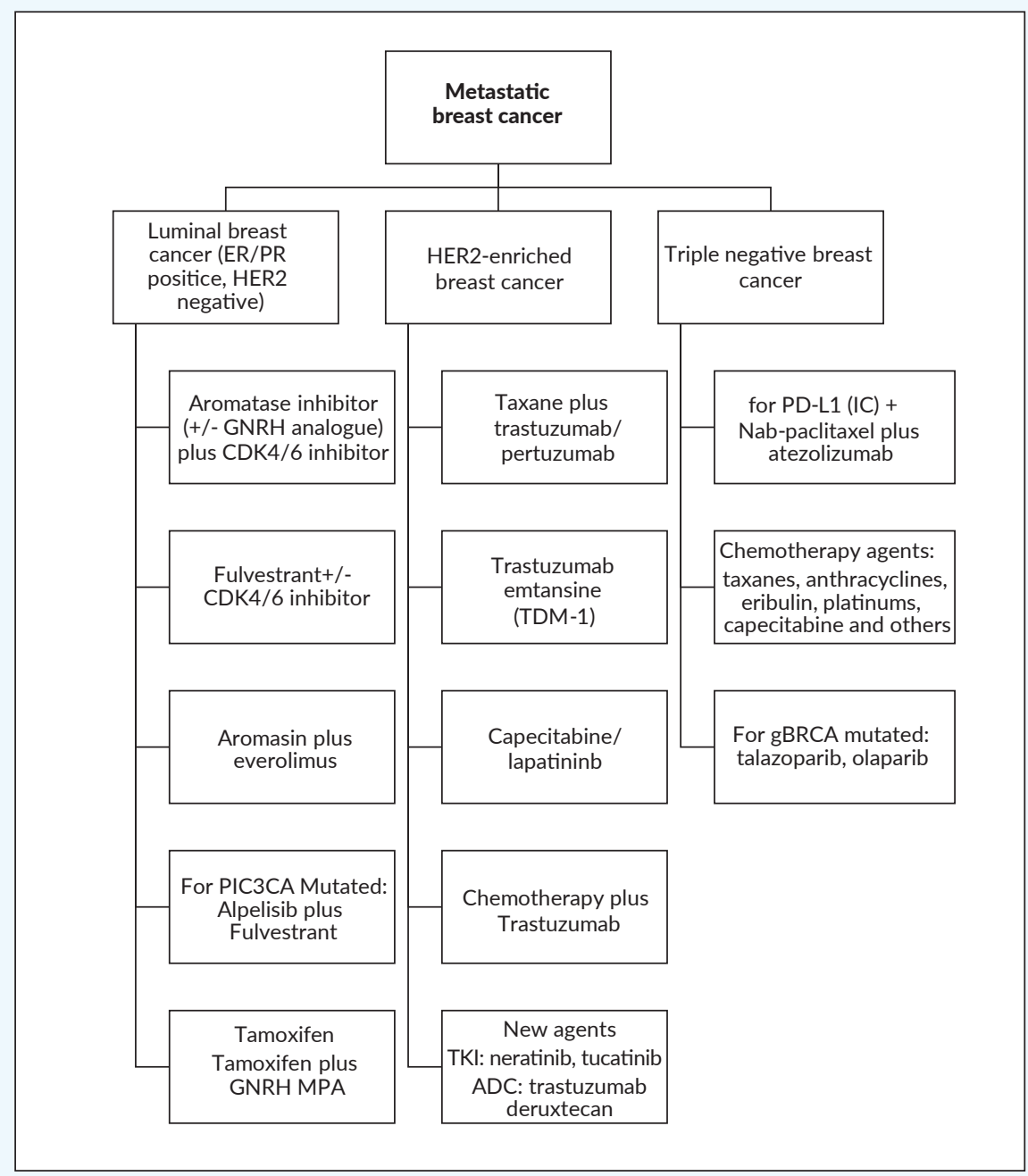

Figure 1. Treatment decisions in the metastatic setting based on intrinsic subtypes.ER, estrogen receptor; HER2, human epidermal growth factor receptor 2, PR, progesterone receptor; GNRH, gonadotropin releasing hormones; CDK, cyclin depended kinase; MPA, medroxy-progestine; TKI, tyrosine kinase inhibitors.

agents have been approved for the use in HER2-positive patients with metastatic breast cancer (Table 4; Figures 1-3). Among patients without $\mathrm{pCR}$, who represent a group with the worst clinical outcomes, trastuzumab emtansine (TDM-1) provided a considerable survival benefit. In this subpopulation, TDM-1 was associated with a 3 -year diseasefree survival of almost $90 \% .{ }^{12}$ In addition to TDM-1, only multimodal therapy, including neoadjuvant chemotherapy with trastuzumab/pertuzumab, optimal surgical approach, radiotherapy and endocrine therapy has resulted in such high survival rates. Therefore, this treatment regimen has led to an optimal treatment with the best available prognosis. ${ }^{8}$

In the metastatic setting, the common standard of care is the combination of pertuzumab plus trastuzumab and docetaxel, based on the CLEOPATRA study. ${ }^{54}$ This trial demonstrated a tremendous OS benefit of 16 months with this triplet combination. In the second-line therapy, the antibody-drug conjugate (ADC) TDM-1 is the standard of care. ${ }^{55}$
Later line options include lapatinib, chemotherapy/ trastuzumab and lapatinib/trastuzumab. Landmark clinical trials investigating these treatment regimens are outlined in Table 4. Recently, other agents including tyrosine kinase inhibitors (TKIs) like neratinib, tucatinib, and pyrotinib, as well as ADCs like trastuzumab deruxtecan showed promising activity. ${ }^{56-58}$

To further optimize treatment in the metastatic setting, several novel strategies have been evaluated in clinical practice and randomized controlled trials (Figure 2). One important strategy seems to be the development of ADC. Trastuzumab deruxtecan is a potent ADC build of a trastuzumab antibody linked with a potent cytotoxic topoisomerase inhibitor. The ADC has a very high payload of chemotherapy with better diffusion agents. ${ }^{59}$ The phase II clinical trial (DESTINYBreast 001) evaluated trastuzumab deruxtecan in patients with HER2-positive metastatic breast cancer who had received a median of 6 prior lines of therapy (range: $2-27$ ), including 
Table 4. Landmark trials in metastatic HER2 positive breast cancer. CL, capecitabine/lapatinib; DFI, disease-free interval; $\mathrm{M}$, months; TH, docetaxel/trastuzumab; DT, docetaxel/trastuzumab; DTP, docetaxel/trastuzumab/pertuzumab; L, lapatinib; OS, overall survival; T-DM1, trastuzumab emtansine; TL, trastuzumab/lapatinib; TPC, treatment of physicians choice.

\begin{tabular}{|c|c|c|c|c|}
\hline & $\begin{array}{l}\text { CLEOPATRA } \\
\qquad(\mathrm{N}=808)\end{array}$ & $\begin{array}{l}2^{\text {nd }} \text { line Emilia } \\
\qquad(\mathrm{N}=991)\end{array}$ & $3^{\text {rd }}$ line+ TH3RESA $(N=602)$ & $\begin{array}{c}3^{\text {rd }} / 4^{\text {th }} \text { line EGF104900 } \\
(N=291)\end{array}$ \\
\hline Drug & $\begin{array}{l}\text { Docetaxel, trastuzumab, } \\
\text { pertuzumab }\end{array}$ & T-DM1 & T-DM1 & Lapatinib \\
\hline Design & DT vs DTP & T-DM1 vs CL & T-DM1 vs TPC & TL vs. L \\
\hline Delta OS & $15.7 \mathrm{M}$ (40.8 vs 56.6$)$ & $4 \mathrm{M} \mathrm{(25.9} \mathrm{vs} 29.9)$ & $6.9 \mathrm{M}(15.8$ vs 22.7$)$ & $4.5 \mathrm{M}$ \\
\hline Side effects & Slightly increased & Better for TDM-1 & Better for TDM-1 & Slightly increased \\
\hline $\begin{array}{l}\text { Prior treatment with } \\
\text { trastuzumab }\end{array}$ & $10 \%$ Interval >12 M & $\begin{array}{l}100 \% \text { (if adjuvant, DFI < } 6 \\
\text { months, } 16 \% \text { of patients) }\end{array}$ & $\begin{array}{l}\text { Prior trastuzumab and } \\
\text { lapatinib }\end{array}$ & $100 \%$ (<3 regimes) \\
\hline Literature & 54 & 55 & 120 & 121 \\
\hline
\end{tabular}

treatment with TDM $1 .^{57}$ In this two-step study, patients received $5.4 \mathrm{mg} / \mathrm{kg}$ trastuzumab deruxtecan every 3 weeks intravenously $(\mathrm{N}=184)$. The response rate was $60.9 \%$, whilst the disease control rate was $97.3 \%$. After a median follow-up of 11.1 months, the median PFS was 16.4 months. Based on this encouraging result, this therapy received FDA approval. Several clinical trials are on the way to determine the role in different clinical settings. ${ }^{60}$

\section{HR-positive breast cancer: Endocrine therapy, CDK4/6 inhibitors and beyond}

Patients with hormonal receptor-positive $(\mathrm{HR}+)$ breast cancer are at increased risk of late relapses after 5 years. ${ }^{61}$ In fact, in high-risk node-positive HR-positive patients with $>3$ lymph nodes, the 5 -year relapse rate is up to $40 \%{ }^{26}$ Tamoxifen was the standard of care in metastatic $\mathrm{HR}+$ breast cancer, as it provided a high clinical benefit rate and a PFS of around 12-15 months in the primary setting. ${ }^{62}$ In premenopausal patients, the addition of gonadotropin-releasing hormone ( $\mathrm{GnRH}$ ) analogs to tamoxifen improved both, response rate and $O S$ in a randomized, prospective clinical trial. ${ }^{63}$ In addition, treatment with $\mathrm{GnRH}$ analogs alone or in combination with tamoxifen leads to recurrence-free survival and OS outcomes comparable with those achieved by different chemotherapy protocols in patients with $\mathrm{HR}+$ breast cancer. $^{62}$ In this patient population, adjuvant therapy with GnRH agonists and tamoxifen is also associated with the preservation of reproductive function..$^{64,65}$

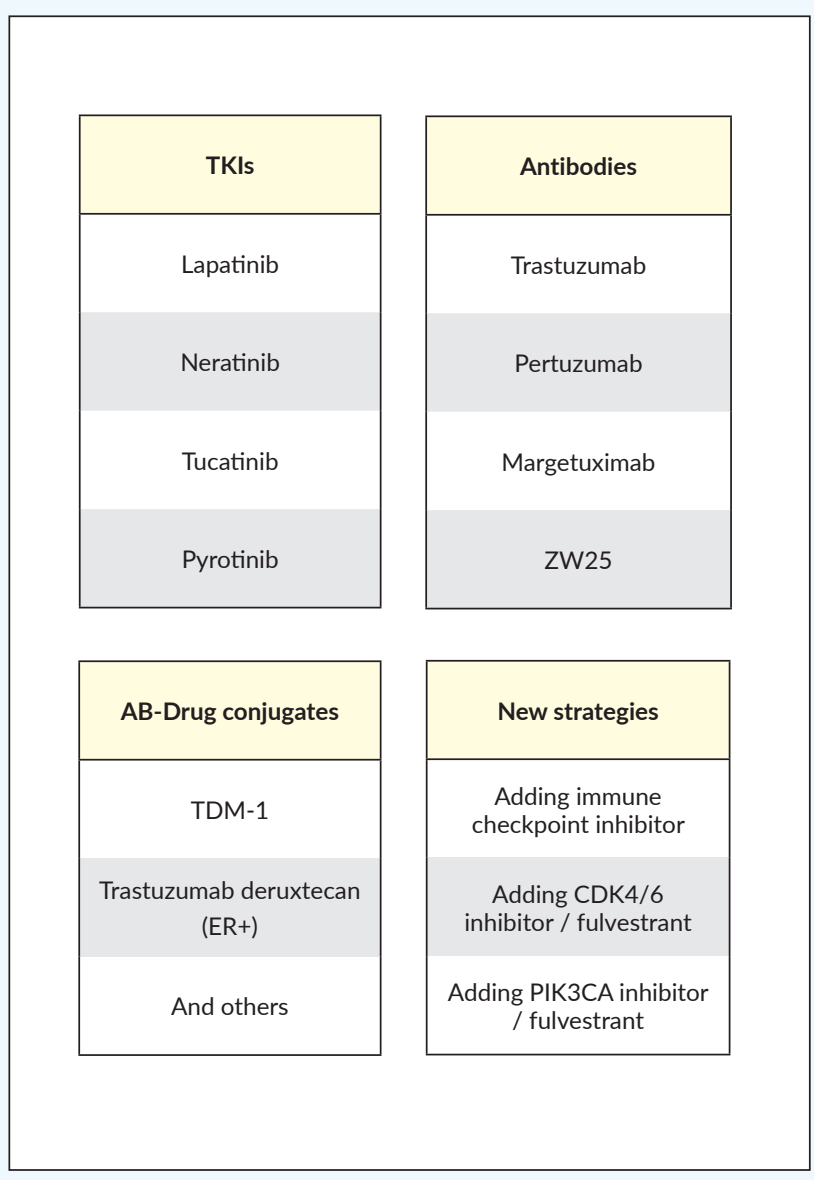

Figure 2. HER2 directed strategies (old and new agents). AB, antibody; ER, estrogen receptor; TDM-1, Trastuzumab Emtansine; TKI, tyrosine kinase inhibitors. 


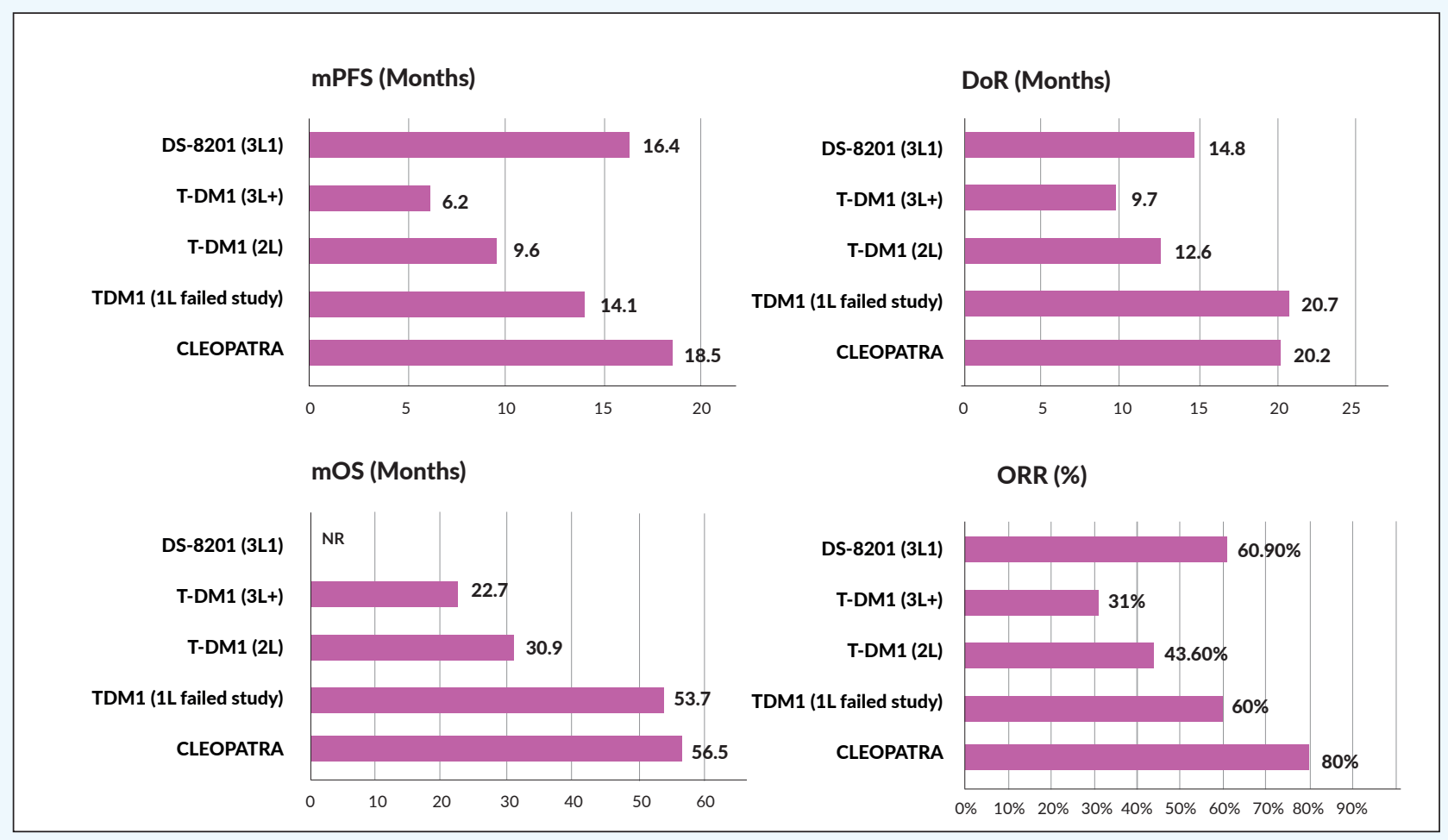

Figure 3. Demonstrated important numbers HER2 directed therapy including new agents (CAVE: no head-to-head comparison). Replace with "DoR, duration of response; mOS, median overall-survival; mPFS, median progression-free-survival; ORR, overall response rate.

Aromatase inhibitors (AI) have been in clinical use for two decades, showing improved clinical outcomes in postmenopausal and premenopausal patients with metastatic breast cancer. ${ }^{66-68}$ However, a high proportion of patients with metastatic disease develops endocrine resistance leading to disease progression within $12-24$ months. $^{69,70,71}$ In the first-line endocrine therapy setting, the clinical benefit rate is around $60 \%$. Subsequent lines have a clinical benefit rate of $40 \%, 24 \%$ and $16 \% .^{72}$ Beyond third-line therapy, AIs are not beneficial in most patients and a switch to chemotherapy is recommended. ${ }^{8}$

Clinically endocrine resistance can be divided into primary and secondary (acquired) type. ${ }^{73}$ Primary endocrine resistance is defined as a relapse less than 2 years after finishing adjuvant endocrine therapy or progression of disease within 6 months on endocrine therapy in the metastatic setting. Secondary resistance is defined as a relapse less than 12 months after finishing endocrine therapy or progression later than 6 months on endocrine therapy for metastatic disease.

The current research has strongly focused on elucidating mechanisms of resistance, and several mechanisms involved in the development of endocrine resistance have been identified. ${ }^{74}$ One of them is the loss of steroidal receptors during disease progression. A Swedish cohort study demonstrated discordance rates in biomarkers between the primary tumor and corresponding relapse ranging from $14 \%$ for ER and 39\% for PR. This study also showed that loss of ER or PR in the relapse was associated with a 3-fold increase of death risk compared with patients who had stable ER- or PRpositive tumors. ${ }^{25}$ Therefore, patients with recurrent disease should receive a biopsy in order to confirm tissue diagnosis and measure the expression of biomarkers, which subsequently determinate the therapy course. ${ }^{75-79}$ In the era of precision medicine and targeted management, the availability of fresh tissue has become increasingly important to determine optimal treatment approach. ${ }^{29}$

A number of approaches have been proposed to overcome the endocrine resistance. One of the strategies is to combine endocrine therapy with targeted agents. ${ }^{80}$ Here, several pathways and alterations involved in breast cancer have been utilized, including alterations and mutations in the ESR 1 gene coding for the estrogen receptors. ${ }^{81-83}$ So far, several ESRI mutations have been described. ${ }^{84,85}$ To overcome the endocrine resistance due to ESR1 mutations, selective estrogen receptor degradation (SERD) drugs have been used for the treatment of patients who failed after AI or tamoxifen therapy. ${ }^{86}$ Fulvestrant, the commonly known SERD, showed promising activity in the advanced endocrine resistant setting. ${ }^{87,88}$

Further resistance mechanisms are caused by amplification and upregulation of co-activators, as well as alterations in 
Table 5. Important trials of HR+ metastatic breast cancer with CDK4/6 inhibitors. Al, aromatase inhibitor, PD, progressive disease; mPFS, median progression-free-survival; mOS, median overall survival; $\mathrm{M}$, months; $\mathrm{NR}$, not reached.

\begin{tabular}{|c|c|c|c|c|c|c|}
\hline Trial & Drug & Population & $\mathrm{N}$ & mPFS & mOS & Literature \\
\hline \multicolumn{7}{|c|}{ Combinations with Al } \\
\hline PALOMA-2 & Palbociclib & Primary therapy & 666 & 24.8 vs $14.5 \mathrm{M}$ & NR & 104 \\
\hline MONALEESA-2 & Ribociclib & Primary therapy & 668 & 25.3 vs $16 \mathrm{M}$ & NR & 106 \\
\hline MONARCH-3 & Abemaciclib & Primary therapy & 493 & 28.1 vs $14.76 \mathrm{M}$ & NR & 109 \\
\hline \multicolumn{7}{|l|}{$\begin{array}{l}\text { Combinations with } \\
\text { fulvestrant }\end{array}$} \\
\hline PALOMA-3 & Palbociclib & $\begin{array}{l}\text { PD on endocrine } \\
\text { therapy }\end{array}$ & 521 & $\begin{array}{l}9.5 \text { vs } 4.6 \mathrm{M} \\
p<0.001\end{array}$ & $\begin{array}{l}34.9 \text { vs } 28 \\
M p=0.09\end{array}$ & 122 \\
\hline MONALEESA-3 & Ribociclib & $\begin{array}{l}0-1 \text { line for metastatic } \\
\text { breast cancer }\end{array}$ & 726 & $\begin{array}{l}20.5 \text { vs } 12.8 \mathrm{M} \\
p<0.001\end{array}$ & $\begin{array}{l}\text { NR vs } 40 \mathrm{M} \\
p=0.005\end{array}$ & 123 \\
\hline MONARCH-2 & Abemaciclib & $\begin{array}{l}\leq 1 \text { line of endocrine } \\
\text { therapy }\end{array}$ & 669 & $\begin{array}{l}16.4 \text { vs } 9.3 \mathrm{M} \\
p<0.001\end{array}$ & $\begin{array}{l}46.7 \text { vs } \\
37.3 \mathrm{M} \\
p=0.014\end{array}$ & 108 \\
\hline
\end{tabular}

co-repressors, e.g., AIB1 and MNAR/PELP1. ${ }^{89-91}$ Promising targets are pathways with a cross-talk to the steroidal hormonal pathway. Most studied alterations involve cyclin-depended kinase 4 and 6 (CDK4/6) proteins such as PIK3CA, ESR1, CCND1, FGFR1, BRCA1, BRCA2, AKT1 and HER2. Several drug combinations are now under investigation. Targeting the PIK3CA pathway seems to be effective, however, most analyses are still experimental and look at a combination of factors, such as mTOR inhibitors. ${ }^{92-94}$ So far, pan-PI3K inhibitors, such as buparlisib, pictilisib, and SAR245408, have not shown impressive efficacy, whereas PI3K- $\alpha$-specific inhibition has shown more promise.

Recently, the alpha-specific PIK3CA inhibitor alpelisib was approved in the metastatic setting in ER+ PIK3CA-mutated metastatic breast cancer. ${ }^{95}$ Data from the SOLAR-1 study demonstrated a substantial PFS benefit in patients treated with alpelisib plus fulvestrant compared with patients treated with placebo plus fulvestrant (11.0 months [95\% CI: 7.5-14.5] vs 5.7 months [95\% CI: 3.7-7.4]). The drug is well-tolerated, with the most common side effects being hyperglycemia, gastrointestinal toxicity and skin toxicity.

Everolimus, an mTOR inhibitor, showed good clinical outcomes in the endocrine resistance setting. ${ }^{96}$ In a large randomized, phase III trial, the combination of everolimus and exemestane led to significantly improved PFS compared with placebo plus exemestane (median PFS, 10.6 months vs 4.1 months; HR: 0.36 [95\% CI: $0.27-0.47$ ]; $\mathrm{p}<0.001$ ) in HR+, HER2- patients with advanced breast cancer with prior exposure to nonsteroidal aromatase inhibitors (NSAIs). Based on the trial results, the drug was approved by the FDA and EMA for patients with metastatic breast cancer that had received previous treatment. Everolimus was also tested in a combination of tamoxifen and fulvestrant, where it showed comparable clinical benefits. ${ }^{97,98}$ Currently, everolimus is being investigated in the adjuvant setting.

Targeting CDKs 4/6, the key regulators of the cell cycle seems to be even more promising. The expression of the $\mathrm{D}$ type cyclin, another important component of the cell cycle, is upregulated by several mitogenic signaling pathways, including steroid hormones (such as the ER pathway), PI3K/AKT/mTOR, MAPKs, WNT/ $\beta$-catenin, STATs, and NF- $\kappa B / I K K .99$ The formation of a complex of cyclin D with CDK4/6 leads to the hyperphosphorylation of the retinoblastoma $(\mathrm{Rb})$ protein, which in turn activates the E2F transcription factors and the progression of the cell cycle. ${ }^{100}$

Currently, three CDK4/6 inhibitors are approved by the FDA: palbociclib, ribociclib, and abemaciclib. ${ }^{101-103}$ The approvals were based on findings from large phase III studies including MONALEESA (ribociclib), PALOMA (palbociclib), and MONARCH (abemaciclib). ${ }^{104-109}$ All studies showed a 
clinical benefit of these three treatment regimen versus the standard of care endocrine therapy, e.g., AI or fulvestrant in both treatment-naïve patients and in those that had relapsed or progressed during prior endocrine therapy. The trials showed significantly improved PFS, with hazard ratios ranging between 0.5 and 0.6 . The most common side effects included neutropenia, although neutropenic fever was rare, with only $1-2 \%$ of all cases. Table 5 provides an overview of the most frequent adverse events associated with the three treatment regimens. Although these drugs are generally well-tolerated, there are some differences in the toxicity profile. Most importantly, however, the combination of the new therapies promises an estimated time of endocrine treatment of more than 36 months before switching to a chemotherapy regime.

\section{Treatment beyond the current classification based on the molecular profile}

Currently, the treatment of early-stage and metastatic breast cancer is based on established, yet simple, molecular markers, including ER, PR, HER2, which have prognostic and predictive value. ${ }^{8}$ Recently, BRCA1/2 and PIK3CA mutational status as well as PD-L1 expression status have been introduced into clinical practice and demonstrated clear clinical benefit for the treatment with olaparib/talazoparib, alpelisib, and atezolizumab. ${ }^{41,110,111}$
Recently, the Breast International Group (BIG) has initiated AURORA, an ongoing, multinational molecular screening program for patients with advanced breast cancer, with the aim to generate new insights in the molecular treatment of breast cancer. ${ }^{112,113}$ In total, around 1,300 patients with metastatic breast cancer ( $\geq 1$ line of systemic treatment for advanced disease), have been planned to be included. In this trial, archival and fresh tissue samples of metastatic lesions and blood are analyzed by NGS for a comprehensive cancer panel of breast cancer-related genes. In a first pilot study using an Ion Torrent sequencing platform at a central facility, 73\% of patients had at least on targetable alteration. Orthogonal validation, which was done by Illumina sequencing technology, resulted in an average of $66 \%$ concordance of substitution calls per patient. Similarly, copy number aberrations obtained from the Ion Torrent sequencing were concordant in 59\% with single nucleotide polymorphism (SNP) arrays, demonstrating that the next-generation genomic techniques are applicable to international molecular screening programs in routine clinical settings. In addition, the primary analysis of the study presented at the ESMO Breast Cancer 2019 congress included molecular results of 381 patients (accrual by November 2017). Among them, pathological subtype distribution was 232 patients with HR+/HER2-, 69 patients with HER2+, 77 patients with TNBC, and 3 with not available subtype. The study identified the presence of subtype

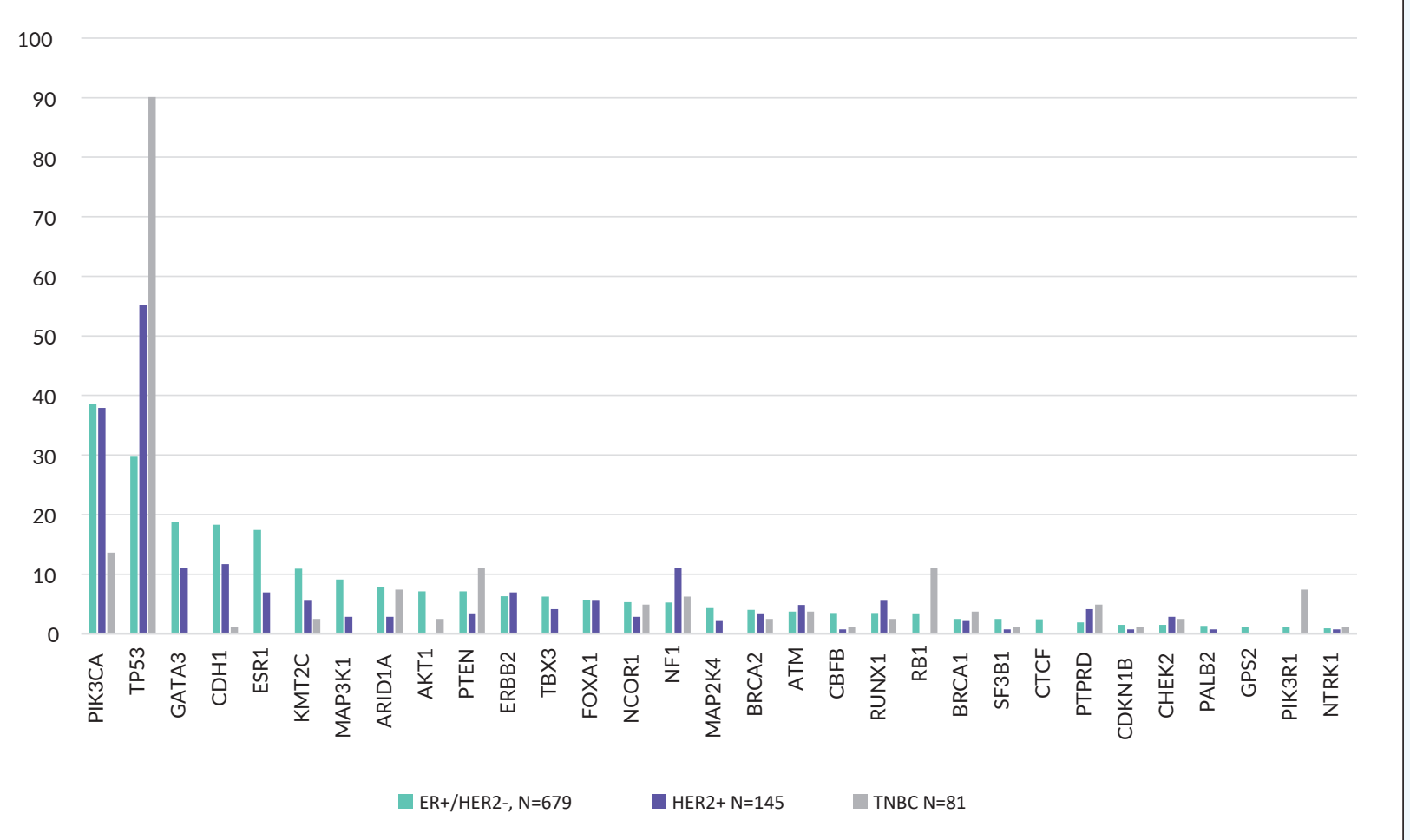

Figure 4. Molecular alterations in metastatic breast cancer (luminal, HER2 and TNBC subtype). Adapted from Razavi et al. $2018 .{ }^{124}$ ER, estrogen receptor; HER2, human epidermal growth factor receptor 2; TNBC, triple-negative breast cancer. 
Table 6. Molecular alterations that are targetable by FDA/EMA approved drugs. Adapted from: OncoKB(https://www.oncokb.org) and ESMO. Cap, capecitabine; mBC, metastatic breast cancer; PFS, progression-free-survival; ORR, overall response rate; OS, overall survival; TPC, treatment of physician's choice, *not yet approved

\begin{tabular}{|c|c|c|c|c|}
\hline Gene & Alteration & Drug & Benefit & Literature \\
\hline ERBB2/HER2 & Amplification & $\begin{array}{l}\text { Tucatinib } \\
\text { +trastuzumab } \\
\text { +capecitabine }\end{array}$ & $\begin{array}{l}\text { PFS and OS benefit in heavily pretreated } \\
\text { HER2-positive metastatic breast cancer } \\
\text { patients }\end{array}$ & 131 \\
\hline ERBB2 & Amplification & Trastuzumab-deruxtecan & High clinical disease control rate & 57 \\
\hline ERBB2 & Amplification & $\begin{array}{l}\text { Capecitabine + } \\
\text { Trastuzumab + Tucatinib }\end{array}$ & $\begin{array}{l}\text { Increase of survival, also in patients with } \\
\text { brain metastasis }\end{array}$ & 58 \\
\hline ERBB2 & Amplification & $\begin{array}{l}\text { Ado-Trastuzumab } \\
\text { Emtansine }\end{array}$ & PFS benefit against TPC & 120 \\
\hline ERBB2 & Amplification & Trastuzumab & $\begin{array}{l}\text { Additional benefit in combination with } \\
\text { docetaxel }\end{array}$ & 52 \\
\hline ERBB2 & Amplification & Lapatinib & In combination with cap, was standard & 125 \\
\hline ERBB2 & Amplification & Neratinib & $\begin{array}{l}\text { NALA trial, slightly better PFS for neratinib/ } \\
\text { cap then lapatinib/cap }\end{array}$ & 56 \\
\hline ERBB2 & Amplification & Pyrotinib & Better PFS/ORR then lapatinib with cap & 126 \\
\hline ERBB2 & Oncogenic mutations & Trastuzumab + lapatinib & $\begin{array}{l}\text { Higher PFS and clinical benefit rate in the } \\
\text { combination arm }\end{array}$ & 127 \\
\hline ESR1 & Oncogenic mutations & AZD9496 or fulvestrant & $\begin{array}{l}\text { AZD9496 = fulvestrant; } \\
\text { combinations are tested }\end{array}$ & 128 \\
\hline PIK3CA & Oncogenic mutations & Fulvestrant + copanlisib & $\begin{array}{l}\text { Potent PIK3CA inhibitor used in B-cell } \\
\text { lymphoma }\end{array}$ & 129 \\
\hline PIK3CA & Oncogenic mutations & Alpelisib + fulvestrant & $\begin{array}{l}\text { Better PFS then fulvestrant alone, approved } \\
\text { by FDA/EMA in } \mathrm{mBC} \text { with PIK3CA mutations }\end{array}$ & 95 \\
\hline PIK3CA & Oncogenic mutations & GDC-0077 & $\begin{array}{l}\text { A study evaluating the efficacy and safety } \\
\text { of GDC-0077 + palbociclib + fulvestrant } \\
\text { vs placebo + palbociclib + fulvestrant in } \\
\text { patients with PIK3CA-mutant hormone } \\
\text { receptor-positive HER2-negative locally } \\
\text { advanced or metastatic breast cancer }\end{array}$ & $\begin{array}{l}\text { Clinical Study } \\
\text { Identifier: NCT } \\
04191499\end{array}$ \\
\hline AKT1 & E17K & Capivasertib* & $\begin{array}{l}\text { Dose finding study of pan-AKT inhibitor in } \\
\text { gynecologic cancer and breast cancer }\end{array}$ & 130 \\
\hline BRCA1/2 & Oncogenic mutations & Olaparib & In $3^{\text {rd }}$ line and beyond better PFS then TPC & 110 \\
\hline BRCA1/2 & Oncogenic mutations & Talazoparib & In $3^{\text {rd }}$ line and beyond better PFS then TPC & 111 \\
\hline
\end{tabular}

switching from primary breast cancer to metastatic breast cancer, along with mutations in the ESR1, PTEN, KAT6A, $M Y C, M D M 4$, and $A K T 3$ genes, and copy number losses in the $R B I$ and $A R I D I A$ genes. ${ }^{114}$

Taken together, in breast cancer, the most frequent mutations with potential clinical relevance include alterations in the $P I K 3 C A$ and ESR 1 genes. Frequent alterations with unknown clinical relevance include amplification in the CCND1 and FGFR1 pathway. In addition, rare alterations with potential clinical relevance include those in the $B R C A 1 / 2, A K T 1$, and $H E R 2$ genes. In this context, several FDA/EMA approved drugs are available for treating patients with metastatic breast cancer (Table 6, online supplementary Table 1; Figure 4). 


\section{CONCLUSION}

Although the treatment armamentarium for breast cancer has considerably expanded, treatment decision is still based on genetic alterations in the $E R, P R$, and HER 2 genes. However, in the era of precision medicine, several new molecular prognostic and predictive markers are available. In the early luminal cancer setting, genetic signatures help to define and guide treatment decisions in terms of chemotherapy. Furthermore, in patients with early HER2-positive breast cancer, clinical markers like pCR mainly direct decisions in clinical practice. In the advanced setting, molecular profiling can guide treatment decisions (e.g., treating PIK3CA-positive patients with a PIK3CA inhibitor) and can help to identify new targets. The most promising pathways included cyclindependent kinase, PIK3CA/mTOR/AKT pathway, BRCA pathway, or overexpression of PD-L1 in patients with TNBC. Further investigation is necessary to standardize the diagnostic and therapeutic approach in breast cancer patients. In the future, every breast cancer patient will have a molecular profile of their tumor to tailor treatment and improve the outcome and toxicity.

\section{TAKE-HOME MESSAGES}

- In the management of breast cancer, a morphologically and genetically heterogeneous disease, estrogen receptor (ER), progesterone receptor (PR), and human epidermal growth factor receptor 2 (HER2) are important predictive factors of response to a given therapy in both, the adjuvant and metastatic setting.

- The recent advancements of molecular profiling methods like next-generation sequencing (NGS), proteomics, and immune profiling have enabled the identification of different actionable targets including alterations in the PI3KCA, ESRI and BRCAI/2 genes as well as programmed death-ligand 1 (PD-L1) expression. Novel targeted therapies have been investigated in clinical trials, with promising results in specific molecular subtypes.

- Several international programs, including AURORA from the Breast International Group (BIG), have been launched to improve our understanding of the pathogenesis of breast cancer and deepen our insights into the molecular treatment of the disease.
1. Siegel RL et al. Cancer statistics, 2019. CA Cancer J Clin. 2019;69(1):7-34

2. Hu Ket al. Global patterns and trends in the breast cancer incidence and mortality according to sociodemographic indices: A observational study based on the global burden of diseases. BM Open. 2019; 9(10): 1-8.

3. Narod SA et al. Why have breast cancer mortality rates declined? J Cancer Policy. Elsevier Ltd.; 2015; 5: 8-17.

4. Bray F et al. Global cancer statistics 2018: GLOBOCAN estimates of incidence and mortality worldwide for 36 cancers in 185 countries. CA Cancer J Clin. 2018; 68(6): 394-424.

5. Montazeri A. Quality of Life in Breast Cancer Patients: A 5. Montazeri A. Quality of Life in Breast Cancer Patients: A Overview of the Literature. In: Preedy VR, Watson RR, editor New York, NY: Springer New York; 2010. p. 2829-55.

New York, NY: Springer New York; 2010. p. 2829-55.
6. Esteva FJ et al. Immunotherapy and targeted therapy combi6. Esteva FJ et al. Immunotherapy and targeted therapy combi-
nations in metastatic breast cancer. Lancet Oncol. 2019; 20(3) nations in
e175-86.

e175-86.
7 . Curtis $\mathrm{C}$ et al. The genomic and transcriptomic architecture

7. Curtis $\mathrm{C}$ et al. The genomic and transcriptomic architecture
of 2,000 breast tumours reveals novel subgroups. Nature. 2012; of 2,000 breast tumo

886(7403): $346-52$. 8. Telli ML et al. NCCN Guider
JNCCN. 2019; 17(5.5): 552-5.

JNCCN. 2019; 17(5.5): 552-5.
9. Schmid P et al. Pembrolizumab for Early Triple-Negative Bre9. Schmid P et al. Pembrolizumab for Early Triple-
ast Cancer. N Engl J Med. 2020; 382(9): 810-21.

10. Perez EA et al. Adjuvant therapy of triple negative breast can cer. Breast Cancer Res Treat. 2010. p. 285-91.

11. Gianni $L$ et al. 5-year analysis of neoadjuvant pertuzuma and trastuzumab in patients with locally advanced, inflammatory, or early-stage HER2-positive breast cancer (NeoSphere): multicentre, open-label, phase 2 randomised trial. Lancet Oncol. 2016; 17(6): 791-800.

12. von Minckwitz G et al. Trastuzumab Emtansine for Residual Invasive HER2-Positive Breast Cancer. N Engl J Med. 2019; $380(7): 617-28$

13. Sparano JA et al. Adjuvant Chemotherapy Guided by a 21 Gene Expression Assay in Breast Cancer. N Engl J Med. 2018; 379(2): 111-21.

14. Cardoso F et al. 70-Gene Signature as an Aid to Treatment Decisions in Early-Stage Breast Cancer. N Engl J Med. 2016 375(8): 717-29.

15. Fitzal $\mathrm{F}$ et al. The genomic expression test EndoPredict is a prognostic tool for identifying risk of local recurrence in postmenopausal endocrine receptor-positive, her2neu-negative breas cancer patients randomised within the prospective ABCSG 8 trial. Br J Cancer. 2015; 112(8): 1405-10.

16. Sestak I et al. Prediction of late distant recurrence after 5 years of endocrine treatment: a combined analysis of patients from the Austrian breast and colorectal cancer study group 8 and arimdex, tamoxifen a lone or in combination randomized trials using the PAM50 risk. J Clin Oncol. 2015; 33(8): 916-22.

17. Citron ML et al. Randomized trial of dose-dense versu conventionally scheduled and sequential versus concurrent combination chemotherapy as postoperative adjuvant treatment of node-positive primary breast cancer: first report of Intergroup Trial C9741/Cancer and Leukemia. J Clin Oncol. 2003; 21(8) $1431-9$

18. Early Breast Cancer Trialists' Collaborative Group 18. Early Breast Cancer Trialists Collaborative Group

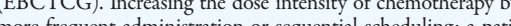
matient-level meta-analysis of 37298 women with early breast cance . Lancet. 2019;393(10179): 1410-52. 19. Schneeweiss A et al. Intense dose-dense epirubicin, paclitaxel, cyclophosphamide versus weekly paclitaxel, liposoma doxorubicin (plus carboplatin in triple-negative breast cancer) for neoadjuvant treatment of high-risk early breast cancer (Ge parOcto-GBG 84): A randomised phase III trial. Eur J Cancer 2019; 106: 181-92

20. Blondeaux E et al. Role of dose-dense chemotherapy in highrisk early breast cancer. Curr Opin Oncol. 2019; 31(6): 480-5. 21. Fumoleau $\mathrm{P}$ et al. Long-term cardiac toxicity after adjuvant epirubicin-based chemotherapy in early breast cancer: French Adjuvant Study Group results. Ann Oncol. 2006; 17(1): 85-92. 22. Kelemen $\mathrm{G}$ et al. Long-term efficiency and toxicity of adjuvant dose-dense sequential adriamycin-Paclitaxel-cyclophosphamide chemotherapy in high-risk breast cancer. Oncology. 2010; 78(3-4): 271-3.

23. Ben-Aharon I et al. Long-Term Follow-Up of Chemotherapy-Induced Ovarian Failure in Young Breast Cancer Patients: The Role of Vascular Toxicity. Oncologist. 2015; 20(9): 985-91. 24. Voduc KD et al. Breast cancer subtypes and the risk of loca and regional relapse. J Clin Oncol. 2010;28(10): 1684-91.

25. Karlsson E et al. Breast cancer during follow-up and progression - A population based cohort on new cancers and changed biology. Eur J Cancer. 2014; 50(17): 2916-24.

26. Pan $\mathrm{H}$ et al. 20-Year Risks of Breast-Cancer Recurrence ofter 26. Pan $\mathrm{H}$ et al. 20-Year Risks of Breast-Cancer Recurrence after Stopping Endocrine $1936-46$

27. Reiter JG et al. Reconstructing metastatic seeding patterns of human cancers. Nat Commun. 2017; 8: 14114 .
28. Zardavas D et al. Clinical management of breast cancer heterogeneity. Nat Rev Clin Oncol. 2015; 12(7): 381-94.

29. Pezo RC et al. Impact of multi-gene mutational profiling on clinical trial outcomes in metastatic breast cancer. Breast Cancer Res Treat. 2017;

30. Bedard PL et al. Tumour heterogeneity in the clinic. Nature. 2013; 501(7467): 355-64.

31. Aurilio $\mathrm{G}$ et al. A meta-analysis of oestrogen receptor, progesterone receptor and human epidermal growth factor receptor 2 discorest 2 discordance between primary b $2014 ; 50(2): 277-89$.

32. Ismail-Khan Ret al. A review of triple-negative breast cancer. Cancer Control. 2010; 17(3): 173-6.

33. Carey L et al Triple-negative breast cancer: disease entity or 33. Carey L et al. Triple-negative breast cancer: disease entity or title of convenience? Nat Rev Clin Oncol. 2010; 7(12): 683-92. 34. Lehmann $\mathrm{BD}$ et al. Identification of human triple-negative
breast cancer subtypes and preclinical models for selection of tarbreast cancer subtypes and preclinical models for selection
geted therapies. J Clin Invest. 2011;121(7):2750-67.

geted therapies. J Clin Invest. 2011; 121(7): 2750-67.
35. Lehmann BD et al. Refinement of Triple-Negative Breast 35. Lehmann BD et al. Refinement of Triple-Negative Breast
Cancer Molecular Subtypes: Implications for Neoadjuvant CheCancer Molecular Subtypes: Implications for Neoad
motherapy Selection. Plos One. 2016; 11(6): 1-22.

36. Lehmann BD et al. Identification and use of biomarkers in 36. Lehmann $\mathrm{BD}$ et al. Identification and use of biomarkers in
treatment strategies for triple-negative breast cancer subtypes. J treatment strategies for triple-n
Pathol. 2014; 232(2): 142-50.

Pathol. 2014; 232(2): 142-50.
37. Caparica R et al. How I treat metastatic triple-negative breast 37. Caparica Ret al. How I treat metastatic triple-
cancer. ESMO open. 2019; 4 (Suppl 2): e000504.

38. Force J et al. Checkpoint Blockade Strategies in the Treatment of Breast Cancer: Where We Are and Where We Are Heading. Curr Treat Options Oncol. 2019; 20(4): 35. 39. Nanda R et al. Pembrolizumab in Patients With Advanced Triple-Negative Breast Cancer: Phase Ib KEYNOTE-012 Study. J Clin Oncol. 2016; 34(21): 2460-7.

40. Voorwerk L et al. Immune induction strategies in metastatic triple-negative breast cancer to enhance the sensitivity to PD-1 blockade: the TONIC trial. Nature medicine. 2019; 25(6): 920-8.

41. Schmid P et al. Atezolizumab and Nab-Paclitaxel in Advanced Triple-Negative Breast Cancer. N Engl J Med. 2018; 379(22): 2108-21.

42. Ahmed FS et al. PD-L1 protein expression on both tumor cells and macrophages are associated with response to neoadjucells and macrophages are associated with response to neoadju-
vant durvalumab in triple negative breast cancer (TNBC). Clin Vancer Res. 2020. [Online ahead of print]

43. Reddy SM et al. Atezolizumab for the treatment of breast cancer. Expert Rev Anticancer Ther. 2020; 20(3): 151-8. 
44. F. Hoffmann-La Roche Ltd. Roche provides update on Phase III study of Tecentriq in combination with paclitaxel for people with metastatic triple-negative breast cancer. 2020 [Accessed September 2020]. Available from: https://www.roche.com/me$\mathrm{dia} /$ releases/med-cor-2020-08-06.htm.

45. Porta Cet al. Targeting PI $3 \mathrm{~K} / \mathrm{Akt} / \mathrm{mTOR}$ Signaling in Cancer. Front Oncol. 2014; 4.

46. Lin Ket al. An ATP-Site On-Off Switch That Restricts Phosphatase Accessibility of Akt. Science Signaling. 2012; 5(223): ra37-ra37.

47. Saura C et al. A First-in-Human Phase I Study of the ATPCompetitive AKT Inhibitor Ipatasertib Demonstrates Robust and Safe Targeting of AKT in Patients with Solid Tumors. Cancer discov. 2017; 7(1): 102-13.

48. Kim S-B et al. Ipatasertib plus paclitaxel versus placebo plus paclitaxel as first-line therapy for metastatic triple-negative breast cancer (LOTUS): a multicentre, randomised, double-blind, placebo-co

49. Schmid P et al. Phase Ib study evaluating a triplet combina49. Schmid P et al. Phase lb study evaluating a triplet combina-
tion of ipatasertib (IPAT), atezolizumab (atezo), and paclitaxel tion of ipatasertib (IPAT), atezolizumab (atezo), and paclitaxel
(PAC) or nab-PAC as first-line (1L) therapy for locally advanced/metastatic triple-negative breast cancer (TNBC) [abstract]. Proceedings of the American Association for Cancer Research Proceedings of the American Association for Cancer Research
Annual Meeting 2019; 2019 Mar 29-Apr 3; Atlanta, GA. PhilAnnual Meeting 2019; 2019 Mar 29-Apr 3; Atlanta, GA. Phil-
adelphia (PA): AACR; Cancer Res 2019;79 (13 Suppl):Abstract $\operatorname{mr}$ CT049.

0. Loibl S et al. HER2-positive breast cancer. Lancet. 2017; 389(10087): 2415-29.

51. Surgery BC. Key words: 2003; 5(September): 649-52.

52. Tedesco KL et al. Docetaxel combined with trastuzumab is an active regimen in HER-2 3+ overexpressing and fluorescent in situ hybridization-positive metastatic breast cancer: a multiinstitutional phase II trial. J Clin Oncol. 2004; 22(6): 1071-7. 53. Schroeder MC et al. Early and Locally Advanced Metaplastic Breast Cancer: Presentation and Survival by Receptor Status in Surveillance, Epidemiology, and End Results (SEER) 20102014. Oncologist. 2018; 23(4): 481-8.

54. Swain SM et al. Pertuzumab, trastuzumab, and docetaxel for HER2-positive metastatic breast cancer (CLEOPATRA): end-of-study results from a double-blind, randomised, placebocontrolled, phase 3 study. Lancet Oncol. 2020; 21(4): 519-30. 55. Diéras $V$ et al. Trastuzumab emtansine versus capecitabine plus lapatinib in patients with previously treated HER2-positive advanced breast cancer (EMILIA): a descriptive analysis of final overall survival results from a randomised, open-label, phase 3 trial. Lancet Oncol. 2017; 18(6): 732-42.

56. Saura C et al. Neratinib Plus Capecitabine Versus Lapatinib Plus Cate Previously Treated With $\geq 2$ HER2-Directed Regimens: Phase III NALA Trial. J Clin Oncol. 2020; 38(27):3138-49.

57. Modi $S$ et al. Trastuzumab Deruxtecan in Previously Trea57. Modi $S$ et al. Trastuzumab Deruxtecan in Previously Trea-
ted HER2-Positive Breast Cancer. N Engl J Med. 2020; 382(7): ted HER2-Po-21.

58. Murthy RK et al. Tucatinib, Trastuzumab, and Capecitabine for HER2-Positive Metastatic Breast Cancer. N Engl J Med. 2020; 382(7): 597-609.

59. Keam SJ. Trastuzumab Deruxtecan: First Approval. Drugs. $2020 ; 80(5): 501-8$

60. Kreutzfeldt $J$ et al. The trastuzumab era: current and upcoming targeted HER2 + breast cancer therapies. Am J Cancer Res. $2020 ; 10(4): 1045-67$

61. Early Breast Cancer Trialists' Collaborative Group (EBCTCG) et al. Aromatase inhibitors versus tamoxifen in early breast cancer: patient-level meta-analysis of the randomised trials. Lancet. 2015; 386(10001): 1341-52.

62. Rugo HS et al. Endocrine therapy for hormone receptor-positive metastatic breast cancer: American society of clinic

63. Klijn JG et al. Combined treatment with buserelin and tamoxifen in premenopausal metastatic breast cancer: a randomized study. J Natl Cancer Inst. 2000; 92(11): 903-11.

64. Franco JG et al. Adjuvant therapy with GnRH agonists/tamoxifen in breast cancer should be a good council for patients with hormone receptor-positive tumours and wis

fertility. Medical Hypotheses. 2012; 78(4): 442-5.
65. Günthert AR et al. Analogs of GnRH-I and GnRH-II inhibit epidermal growth factor-induced signal transduction and resensitize resistant human breast cancer cells to $4 \mathrm{OH}$-tamoxifen. Eur J Endocrinol. 2005; 153(4): 613-25.

66. Gibson L et al. Aromatase inhibitors for treatment of advan66. Gibson L et al. Aromatase inhibitors for treatment of advan-
ced breast cancer in postmenopausal women. Cochrane Dataced breast cancer in postmenopausal wo

67. Bradley R et al. Aromatase inhibitors versus tamoxifen in early breast cancer: Patient-level meta-analysis of the randomised rials. Lancet. 2015:386(10001): 1341-52

68. Mouridsen $\mathrm{H}$ et al. Phase III study of letrozole versus tamoxifen as first-line therapy of advanced breast cancer in postmenopausal women: analysis of survival and update of efficacy
from the International Letrozole Breast Cancer Group. J of Clin Oncol. United States; 2003; 21(11): 2101-9.

69. Sainsbury R. The development of endocrine therapy for women with breast cancer. Cancer Treat Rev. 2013. p. 507-17. 70. Palmieri $\mathrm{C}$ et al. Breast cancer: Current and future endocrine therapies. Mol Cell Endocrinol. 2014. p. 695-723. 71. Ali $S$ et al. ENDOCRINE-RESPONSIVE BREAST CANCER AND STRATEGIES FOR COMBATING RESISTANCE. Nat Rev Cancer. 2002; 2(2): 101-12.

72. Iwase $\mathrm{H}$ et al. Clinical benefit of sequential use of endocrine therapies for metastatic breast cancer. Int J Clin Oncol. 2015;
20(2): 253-61

73. Senkus E et al. Primary breast cancer: ESMO Clinical Practice Guidelines for diagnosis, treatment and follow-up. Ann Oncol. 2013; 24 Suppl 6: vi7-23.

74. Tryfonidis $\mathrm{K}$ et al. Endocrine treatment in breast cance: Cure, resistance and beyond. Cancer Treat Rev. 2016; 50: 68-8 75. Chen $\mathrm{L}$ et al. Trends in 5-year survival rates among breas cancer patients by hormone receptor status and stage. Breast Cancer Res Treat. 2014; 147(3): 609-16.

76. Bae SY et al. Poor prognosis of single hormone receptorpositive breast cancer: similar outcome as triple-negative breast cancer. BMC Cancer. 2015; 15(1): 138

77. Millar EKA et al. Prediction of outcome of early ER breast cancer is improved using a biomarker panel, which includes $\mathrm{K}_{\mathrm{i}}$ 67 and $\mathrm{p} 53$. Br J Cancer. 2011; 105(2): 272-80.

78. Subik $\mathrm{K}$ et al. The expression patterns of ER, PR, HER2 CK5/6, EGFR, KI-67 and AR by immunohistochemical analysis in breast cancer cell lines. Breast Cancer (Auckl). 2010; 4(1) $35-41$.

79. Onitilo AA et al. Breast cancer subtypes based on ER/PR and Her2 expression: Comparison of clinicopathologic features and survival. Clin Med Res. 2009; 7(1-2): 4-13.

80. Mohamed A et al. Targeted therapy for breast cancer. Am J

1. Robinson DR et al. Activating ESR1 mutations in hormone-resistant metastatic breast cancer. Nat Genet. 2013; 45(12): $1446-51$

82. Oesterreich $S$ et al. The search for ESR1 mutations in breast cancer. Nat Genet. 2013; 45(12): 1415-6.

83. Holst $\mathrm{F}$ et al. Estrogen receptor alpha (ESR1) gene amplification is frequent in breast cancer. Nat Genet. 2007; 39(5)

84. Jia $S$ et al. Clinically Observed Estrogen Receptor Alpha Mutations within the Ligand-Binding Domain Confer Distinguishable Phenotypes. Oncology. 2018; 94 (3): 176-89.

85. Jeselsohn R. Are We Ready to Use ESR1 Mutations in Clincal Practice? Breast Care (Basel). 2017; 12(5): 309-13 86. Xiong R et al. Novel Selective Estrogen Receptor Downregulators (SERDs) Developed against Treatment-Resistant Breast Cancer. J Med Chem. 2017; 60(4): 1325-42.

87. Howel A et al. Fulvestrant versus Anastrozole for the Treatment of Advanced Breast Carcinoma in Postmenopausal Women A Prospective Combined Analysis of Two Multicente Trials. Cancer. 2005; 104(2):236-9

88. Di Leo A et al. Final overall survival: fulvestrant $500 \mathrm{mg}$ vs $50 \mathrm{mg}$ in the randomized CONFIRM trial. J Natl Cancer Inst. 2014; 106(1): djt337.

89. Alkner S et al. The role of AIB1 and PAX2 in primary breast cancer: validation of AIB1 as a negative prognostic factor. Ann Oncol. 2013; 24(5): 1244-52.

90. Girard BJ et al. PELP1: a review of PELP1 interaction signaling, and biology. Mol Cell Endocrinol. 2014; 382(1) $642-51$.

91. Rajhans $R$ et al. Comprehensive analysis of recent biochem $\mathrm{cal}$ and biologic findings regarding a newly discovered proteinPELP1/MNAR. Clin Exp Metastasis. 2006:23(1): 1-7.

92. Janku F et al. PIK3CA Mutations in Patients with Advanced Cancers Treated with PI3K/AKT/mTOR Axis Inhibitors. Mol Cancer Ther. 2011; 10(3): 558-65.

93. Janku F et al. PI3K/AKT/mTOR inhibitors in patients with breast and gynecologic malignancies harboring PIK3CA mutations. J Clin Oncol. 2012; 30(8): 777-82.

94. Janku F et al. PIK3CA mutation H1047R is associated with response to $\mathrm{PI} 3 \mathrm{~K} / \mathrm{AKT} / \mathrm{mTOR}$ signaling pathway inhibitors in early-phase clinical trials. Cancer Research. 2013; 73(1):276-84. 95. André $\mathrm{F}$ et al. Alpelisib for PIK3CA-Mutated, Hormone Receptor-Positive Advanced Breast Cancer. N Engl J Med. 2019; 380(20): 1929-40

96. Piccart $\mathrm{M}$ et al. Everolimus plus exemestane for hormone-receptor-positive, human epidermal growth factor receptor-2-neLERO-2†. Ann Oncol. 2014; 25(12): 2357-62.

97. Massarweh S et al. A phase II study of combined fulvestrant and everolimus in patients with metastatic estrogen receptor (ER)-positive breast cancer after aromatase inhibitor (AI) failure. Breast Cancer Res Treat. 2014; 143(2): 325-32.

98. Bachelot $\mathrm{T}$ et al. Randomized phase II trial of everolimus in combination with tamoxifen in patients with hormone receptorpositive, human epidermal growth factor receptor 2-negative metastatic breast cancer with prior exposure to aromatase inhimetastatic breast cancer with prior exposure to aromatase inhi-
bitors: a GINECO study. J Clin Oncol. 2012; 30(22): 2718-24. 99. Lange $\mathrm{CA}$ et al. Killing the second messenger: targeting loss 99. Lange $\mathrm{CA}$ et al. Killing the second messenger: targeting loss
of cell cycle control in endocrine-resistant breast cancer. Endocr of cell cycle control in endocrine-res

Relat Cancer. 2011; 18(4): C19-24.
100. Finn RS et al. PD 0332991, a selective cyclin D kinase 4/6 100. Finn RS et al. PD 0332991 , a selective cyclin D kinase $4 / 6$ inhibitor, preferentially inhibits proliferation of luminal estrogen receptor-positive human breast
Cancer Res. 2009; 11(5): R77.

101. Gnant $\mathrm{M}$ et al. CDK4/6 inhibitors in luminal breast cancer. 101. Gnant M et al. CDK4/6
Lancet Oncol. 2015. p. 2-3.

Lancet Oncol. 2015. p. 2-3.
102. Hamilton E et al. Targeting CDK4/6 in patients with can102. Hamilton E et al. Targeting CDK4/6
cer. Cancer Treat Rev. 2016. p. 129-38.

cer. Cancer Treat Rev. 2016. p. 129-38.
103. Malumbres M. CDK4/6 Inhibitors resTORe Therapeutic Sensitivity in HER2+Breast Cancer. Cancer Cell. 2016. p. 243-4.

104. Finn RS et al. Palbociclib and Letrozole in Advanced Breast Cancer. N Engl J Med. 2016; 375(20): 1925-36.

105. Turner NC et al. Palbociclib in Hormone-Receptor-Positive Advanced Breast Cancer. N Engl J Med. 2015; 373(3): 209-19.
106. Hortobagyi GN et al. Ribociclib as First-Line Therapy for HR-Positive, Advanced Breast Cancer. N Engl J Med. 2016 375(18): $1738-48$

107. Curigliano $\mathrm{G}$ et al. Ribociclib plus letrozole in early breas cancer: A presurgical, window-of-opportunity study. Breast. 2016; 28: 191-8.

108. Sledge GW et al. MONARCH 2: Abemaciclib in Combination With Fulvestrant in Women With HR+/HER2- Advanced Breast Cancer Who Had Progressed While Receiving Endocrine Therapy. J Clin Oncol. 2017; 35(25): 2875-84.

109. Goetz MP et al. MONARCH 3: Abemaciclib As Initial Therapy for Advanced Breast Cancer. J Clin Oncol. 2017; 35(32): 3638-46.

110. Robson $M$ et al. Olaparib for Metastatic Breast Cancer in Patients with a Germline BRCA Mutation. N Engl J Med 2017; 377(6): 523-33.

111. Litton JK et al. Talazoparib in Patients with Advanced Breast Cancer and a Germline BRCA Mutation. N Engl J Med. 2018; 379(8): 753-63.

112. Maetens $M$ et al. The AURORA pilot study for molecular 112. Maetens $\mathrm{M}$ et al. The AURORA pilot study for molecular
screening of patients with advanced breast cancer-a study of the breast international group. NPJ Breast Cancer. 2017; 3: 23.

113. Zardavas D et al. The AURORA initiative for metastatic
. 113. Zardavas D et al. The AURORA initiative
breast cancer. Br J Cancer. 2014; 111(10): 1881-7.

114. Aftimos PG et al. First report of AURORA, the Breast International Group (BIG) molecular screening initiative for metastatic breast cancer (MBC) patients (pts). Ann Oncol (2019) 30 (suppl_3): iii47-iii64.10.1093/annonc/mdz100.

115. Harbeck N et al. St. Gallen 2007: Breast cancer consensus report. Breast Care. 2007; 2(3): 130-4.

116. Nanda R et al. Pembrolizumab in Patients With Advanced Triple-Negative Breast Cancer: Phase Ib KEYNOTE-012 Study. J Clin Oncol. 2016; 34(21): 2460-7

117. Adams $S$ et al. Pembrolizumab monotherapy for previously treated metastatic triple-negative breast cancer: Cohort A of
the phase II KEYNOTE-086 study. Ann Oncol. 2019; 30(3): 397-404. 118. Dirix LY et al. Avelumab, an anti-PD-L1 antibody, in pa-
tients with locally advanced or metastatic breast cancer: a phase
1b JAVELIN Solid Tumor study. Breast Cancer Res Treat. 2018; 1b JAVELIN Solid Tumor study. Breast Cancer Res Treat. 2018 167(3): 671-86

119. Emens LA et al. Long-term Clinical Outcomes and Biomarker Analyses of Atezolizumab Therapy for Patients With Metastatic Triple-Negative Breast

120. Krop IE et al. Trastuzumab emtansine versus treatment of physician's choice for pretreated HER2-positive advanced breat cancer (TH3RESA): a randomised, open-label, phase 3 trial. Lancet Oncol. 2014; 15(7): 689-99.

121. Blackwell KL et al. Overall survival benefit with lapatinib in 121. Blackwell KL et al. Overall survival benefit with lapatinib in
combination with trastuzumab for patients with human epidermal growth factor receptor 2-positive metastatic breast cancer: final results from the EGF104900 Study. J Clin Oncol. 2012; 30(21): 2585-92.

122. Turner NC et al. Overall Survival with Palbociclib and Fulvestrant in Advanced Breast Cancer. N Engl J Med. 2018 26-36.

123. Slamon DJ et al. Phase III Randomized Study of Ribociclib and Fulvestrant in Hormone Receptor-Positive, Human Epidermal Growth Factor Receptor 2-Negative Advanced Breast Cancer: MONALEESA-3.J Clin Oncol. 2018; 36(24): 2465-72.
124. Razavi P et al. The Genomic Landscape of Endocrine-Re124. Razavi $P$ et al. The Genomic Landscape of Endocrine-Re-
sistant Advanced Breast Cancers. Cancer Cell. 2018; $34(3)$ sistant Advance $427-438$.

427-438.e6.
125. Geyer CE et al. Lapatinib plus capecitabine for HER2 positive advanced breast cancer. N Engl J Med. 2006; 355(26) 2733-43.

126. Ma F et al. Pyrotinib or Lapatinib Combined With Capecitabine in HER2-Positive Metastatic Breast Cancer With Prior Taxanes, Anthracyclines, and/or Trastuzumab: A Randomized, Phase II Study. J Clin Oncol. 2019; 37(29): 2610-9.

127. Blackwell KL et al. Randomized study of Lapatinib alone or in combination with trastuzumab in women with ErbB2-poSitive, trastuzumab-refractory

128. Robertson JFR et al. A Randomized, Open-label, Presurgical, Window-of-Opportunity Study Comparing the Pharmacodynamic Effects of the Novel Oral SERD AZD9496 with Fulvestrant in Patients with Newly Diagnosed ER+ HER2- Primary Breast Cancer. Clin Cancer Res. 2020; 26(16):4242-49.

129. Curigliano G et al. Safety and Tolerability of Phosphatidy-
areast Cancer. Clin Cancer Res. 2020; $26(16): 4242-49$. 129. Curigliano G et al. Safety and Tolerability of Phosphatidy-
linositol-3-Kinase (PI3K) Inhibitors in Oncology. Drug Safety. 2019; 42(2): 247-62

130. Jones RH et al. Fulvestrant plus capivasertib versus placebo after relapse or progression on an aromatase inhibitor in metastatic, oestrogen receptor-positive breast cancer (FAKTION): a
multicentre, randomised, controlled, phase 2 trial. Lancet Oncol multicentre, randomised,
$2020 \mathrm{Mar} ; 21(3): 345-57$

131. Murthy RK et al. Tucatinib, Trastuzumab, and Capecitabi2020 Feb 13;382(7):597-60

Online supplementary Table 1

Available from:

https://cdn.healthbook.network/wp-content/uploads/2020/10/table6.jpg 